\title{
STI - Lógica Livre: uma ferramenta para auxiliar a aprendizagem de equivalência lógica no âmbito da educação superior
}

\author{
Marcos A. Schreiner, Fabio H. Gil, Eliana S. Lisbôa, Daniel A. Karling \\ Setor Palotina - Universidade Federal do Paraná (UFPR) \\ Rua Pioneiro, 2153, Jardim Dallas, CEP: 85950-000, Palotina, PR. \\ \{marcosantonio, eliana.lisboa, daniel.karling\}@ufpr.br, \\ fabio.henrique.gilegmail.com
}

\begin{abstract}
The present work deals with the development and evaluation of an Intelligent Tutoring System (ITS) used to aid in the learning of Propositional Logical Equivalence in the Licenciatura em Computação of the Setor Palotina of UFPR.In the development of the ITS, we used Web Development and Artificial Intelligence (IA) techniques, such as Bootstrap and Fuzzy Logic. Its use was based on the solution of challenges by the student, and with each challenge the system reports feedback and accompanies him in the progress of his learning. The software was submitted to experiments, in order to validate it as an educational tool. We had results that show an improvement in students' learning in the Logic discipline of our institution.
\end{abstract}

Resumo. O presente trabalho aborda o desenvolvimento e a avaliação de um Sistema Tutor Inteligente (STI) utilizado para auxiliar na aprendizagem de Equivalência Lógica Proposicional no Curso de Licenciatura em Computação do Setor Palotina da UFPR. Na criação do STI foram utilizadas técnicas de Desenvolvimento Web e de Inteligência Artificial (IA), tais como Bootstrap e Lógica Fuzzy. Sua utilização foi baseada na solução de desafios pelo aluno sendo que, a cada desafio, o sistema lhe reporta um feedback e o acompanha no progresso de sua aprendizagem. O software foi submetido a experimentos, com o intuito de validá-lo enquanto ferramenta educacional. Como resultado obtivemos uma melhora na aprendizagem dos alunos da disciplina de Lógica de nossa instituição.

\section{Introdução}

No contexto atual, permeado por tecnologias digitais, surge o grande interesse de adolescentes e jovens pelas novidades tecnológicas. Um desafio é apresentado aos professores, os quais precisam lidar com uma geração que necessita de maiores estímulos para aprender (Veen e Vrakking, 2009) além de ter que atentar-se às competências e estilos de aprendizagem diferenciados em uma mesma sala de aula (Pimentel, de França, e Omar, 2003).

O modo com que cada aluno adquire conhecimento constitui uma temática de estudos de várias áreas, tais como a Ciência da Computação, a Educação e a Psicologia (Nwana, 1990). Os estudos sobre a aprendizagem individual foram, inclusive, base 
VIII Congresso Brasileiro de Informática na Educação (CBIE 2019)

Anais do XXX Simpósio Brasileiro de Informática na Educação (SBIE 2019)

para o desenvolvimento do clássico Sistema Tutor Inteligente (STI) Scholar ${ }^{1}$ (Carbonell, 1970). Os STIs são softwares que possuem um conhecimento do domínio a ser ensinado e um modelo de aprendizagem do aluno, buscando direcionar o ensino de acordo com as suas características individuais. Conforme Elsom-Cook (1987) a vantagem preliminar de um STI é a possibilidade de fornecer um ensino um-para-um ${ }^{2}$. Tal método é dificilmente alcançado em uma sala de aula, uma vez que um professor ministra suas disciplinas para vários alunos, com diversidades de pensamentos e com vários níveis de conhecimento, ao mesmo tempo.

No caso específico do nível superior, as diferenças que os alunos apresentam entre os conhecimentos prévios parecem ter impacto forte uma vez que, dependendo da disciplina, pode ser gerada para alguns uma dificuldade maior em adquirirem novos conhecimentos. Como exemplo, citamos a disciplina "Lógica Matemática", ministrada nos cursos de Computação de nível superior, na qual muitos alunos têm dificuldades em compreendê-la (Grivokostopoulou, Perikos, e Hatzilygeroudis, 2013; Lodder, Passier, e Stuurman, 2008).

Tomando esta disciplina como exemplo, os discentes do Curso de Licenciatura em Computação da Universidade Federal do Paraná (UFPR) - Setor Palotina, demonstram uma certa dificuldade em aprender seus conteúdos abstratos. Um desses conteúdos é o de "Equivalência Lógica Proposicional", o qual é fundamental para a compreensão do método de dedução natural e de resolução.

Ao perceber as dificuldades e a importância existentes nos conteúdos de lógica do ensino superior, mais especificamente no curso de Licenciatura em Computação da UFPR - Setor Palotina, nos propomos a desenvolver e utilizar um STI, denominado STI - Lógica Livre $^{3}$, com o propósito de verificar, na prática, se esta de fato é uma ferramenta eficaz para minimizar as dificuldades de aprendizagem dos alunos no conteúdo de Equivalência Lógica Proposicional.

Deste modo descreveremos com mais detalhes o trabalho nas próximas seções, subdivididas em: (i) apresentação da teoria de aprendizagem utilizada; (ii) conceituação teóricos de STI; (iii) estrutura e as funcionalidades do STI - Lógica Livre; e (iv) estudo, análise e as discussões sobre os experimentos realizados.

\section{Teoria de Aprendizagem Utilizada}

A teoria subjacente a construção do STI - Lógica Livre foi a Teoria Sócio interacionista de Vygotsky (1978). Essa teoria preconiza que o desenvolvimento do conhecimento tem como alicerce as interações sociais. Para o autor, o desenvolvimento das pessoas ocorrem primeiramente no plano exterior (social) para, em seguida, ser internalizado pelo indivíduo (individual).

Sob essas perspectiva, o indivíduo nasce com potencialidades de aprendizado diferenciadas dos outros animais mas ,para que sejam desenvolvidas, é necessário haver uma mediação social. Outro ponto relevante de sua teoria é o conceito de Zona de Desenvolvimento Proximal (ZDP) e Zona de Desenvolvimento Real (ZDR). A primeira representa

\footnotetext{
${ }^{1}$ Sistema Tutor Inteligente desenvolvido para o ensino de geografia.

${ }^{2}$ Ensino personalizado, onde cada aluno tem seu professor que ensina conforme suas necessidades.

${ }^{3}$ Disponível em: http://lcp.c3sl.ufpr.br/logica_livre/
} 
VIII Congresso Brasileiro de Informática na Educação (CBIE 2019)

Anais do XXX Simpósio Brasileiro de Informática na Educação (SBIE 2019)

a diferença entre a capacidade do indivíduo resolver problemas por si próprio e a capacidade de resolvê-los com ajuda de alguém. Já a segunda abrange todas as funções e atividades que o indivíduo consegue desempenhar por seus próprios meios (Vygotsky, 1978). Ou seja, ZDR é o conteúdo que o aluno domina e ZDP é o conhecimento que ele é capaz de construir mas com o auxílio de outrem.

Deste modo, as atividades de aprendizagem precisam ser planejadas tendo em vista propiciar a colaboração e, o mais importante, elas têm que ser coerentes, tendo como parâmetro a ZDP dos alunos. No caso específico do STI desenvolvido, a proposta incluiu trabalhar o ensino de Lógica na ZDP do aluno utilizando, inicialmente, conceitos básicos para que se possa evoluir por meio da interação com o Tutor.

\section{Sistemas Tutores Inteligentes e Trabalhos Relacionados}

Os STI são "programas de computador com propósitos educacionais e que incorporam técnicas de Inteligência Artificial" (Fowler, 1991) como, por exemplo, as técnicas de representação do conhecimento. Este sistemas podem ser construídos com base na Arquitetura Tripartida, a qual contém: (i) um módulo para representar o conhecimento do aluno (Módulo do Aluno); (ii) um módulo que tem o conhecimento de todos os conteúdos a serem ensinados (Módulo Domínio); e um módulo que responsabiliza-se pelas tomadas de decisões no ensino (Módulo Tutor) (Carbonell, 1970, 1971). Estes três componentes trocam informações que podem favorecer o processo de aprendizagem do aluno, usuário do sistema.

A representação computacional do conhecimento pode ser feita por meio de predicados com o uso da linguagem Prolog. Os predicados são tratadas de forma discreta, sendo verdadeiros (1) ou falsos (0). Entretanto, quando há necessidade de representar incertezas é crucial considerar verdades parciais, que podem ser expressas no intervalo [0,1]. A Lógica Fuzzy, ou difusa, permite representar conhecimentos sobre informações imprecisas ou ambíguas, como por exemplo, "muito", "pouco", "pequeno"e "grande" (Klir e Yuan, 1995).

$\mathrm{Na}$ Lógica difusa os conjuntos são expressos por meio de funções e um elemento pode pertencer a mais de um conjunto ao mesmo tempo. Para isso é calculado um grau de pertinência dos elementos em cada conjunto, cujo valor está contido no intervalo $[0,1]$ (Coppin, 2010). Por exemplo, se a nota de um aluno for 92, ela pode pertencer ao conjunto das notas "altas"e "muito altas", dependendo da função que representa estes dois conjuntos.

Deste modo o processo de inferência da Lógica Fuzzy é dividido em duas etapas: Fuzzificação e Defuzzificação. Na primeira é calculado os valores difusos de todos os elementos a partir dos dados de entrada, da função de representação dos conjuntos fuzzy das regras de inferência. Na segunda, a partir dos valores difusos dos elementos é obtido os valores nítidos ${ }^{4}$, ou seja, as conclusões das regras de inferência (Coppin, 2010).

Avaliar se um aluno domina um determinado assunto é uma tarefa que deve considerar incertezas e sua modelagem por meio de um método discreto nem sempre é viável. Para o STI ser capaz de analisar o processo de aprendizagem do aluno é possível utilizar Lógica Fuzzy. Por exemplo no STI desenvolvido por Rissoli, Giraffa, e Martins (2006)

\footnotetext{
${ }^{4}$ Geralmente nomeados como substantivos, compreensíveis na linguagem natural humana.
} 
VIII Congresso Brasileiro de Informática na Educação (CBIE 2019)

Anais do XXX Simpósio Brasileiro de Informática na Educação (SBIE 2019)

a Lógica Fuzzy foi utilizada para acompanhar a evolução do aprendiz acerca do conteúdo apresentado, a fim de propiciar uma aprendizagem significativa (Ausubel, 2003).

Outro exemplo de STI é o FOL Equivalence System (First-Order Logic Equivalence System) (Grivokostopoulou et al., 2013), um sistema desktop que objetiva abordar a representação de conhecimento e raciocínio mediante uso da Lógica de Primeira Ordem e Linguagem Natural (NL). É possibilitado, ao aluno, selecionar uma fórmula e convertê-la para uma outra equivalente. A cada passo realizado é possível recorrer a um feedback baseado em suas ações e/ou seu estado de conhecimento.

Já o IDEAS (Lodder et al., 2008) desenvolvido na Open University Netherlands é um projeto que consistiu em desenvolver uma ferramenta suporte no ensino de lógica, reescrevendo fórmulas lógicas na Forma Normal Disjuntiva (FND). A ferramenta possibilita a aplicação de regras simples, por parte do aluno, e analisa se a expressão submetida é uma derivação da previamente fornecida, além de fornecer, a cada passo, um feedback novo ao aluno.

O LogEx é outro Sistema Tutor Inteligente, disponível em uma plataforma $W e b^{5}$, que contém três modalidades de exercícios que o aluno pode realizar: (i) reescrita de fórmulas lógicas na Forma Normal Disjuntiva (FND); (ii) reescrita de fórmulas lógicas na Forma Normal Conjuntiva (FNC); e (iii) prova de equivalência entre duas fórmulas.

O LogEx detecta pequenos erros semânticos como, por exemplo, se o aluno tem expressão $\neg(p \vee q)$ e digita $(\neg p \vee \neg q)$, consequentemente o STI compreende que ele tentou aplicar a regra De Morgan e apresenta uma dica: "Quando se aplica a regra de De Morgan, uma disjunção é transformada em uma conjunção" 6 (Lodder, Heeren, e Jeuring, 2015). Se nenhuma regra for detectada, o LogEx utiliza de um conjunto de erros baseados na regra Buggy $y^{7}$ com objetivo de oferecer um feedback apropriado. Também foi implementada a técnica de feed forward, a qual sugere dicas para ajudar o estudante a continuar resolvendo o exercício Lodder, Heeren, e Jeuring (2016).

Por fim, o STI denominado Deep Thought (Mostafavi e Barnes, 2017) utiliza uma modelagem Orientada a Dados $(\mathrm{OD})^{8}$ para realizar seus feedbacks, além de realizar Mineração de Dados (MD) ${ }^{9}$ para verificar o agrupamento entre os alunos. Assim, não somente considera a individualidade dos alunos mas também infere estratégias educacionais através da similaridade com os demais utilizadores do sistema.

A necessidade de melhorias no feedback é um problema destacado nestes estudos. As pesquisas acerca deste tema ainda não se esgotaram. O conteúdo de Equivalência Lógica Proposicional, essencial para o entendimento de Lógica, pode ser transmitido de uma forma melhor. Neste trabalho propomos abordar este conteúdo por meio de desafios, onde o aluno precisa identificar ou utilizar um Regra de Equivalência para resolvê-lo, como veremos na seção a seguir.

\footnotetext{
${ }^{5}$ urlhttp://ideas.cs.uu.nl/logex/

${ }^{6}$ when applying DeMorgan's rule, a disjunction is transformed into a conjunction (Tradução literal).

${ }^{7}$ Conhecido como modelo de perturbação, pois assume que os erros do aluno são decorrentes da concepção errônea de algum conceito ou ausência dele (Gavidia e ANDRADE, 2003)

${ }^{8}$ Leva em conta os dados dos alunos anteriores, realizando agrupamentos de alunos por característica das respostas.

${ }^{9}$ Técnica utilizada para encontrar anomalias, padrões, realizar agrupamento ou classificação dos dados.
} 
VIII Congresso Brasileiro de Informática na Educação (CBIE 2019)

Anais do XXX Simpósio Brasileiro de Informática na Educação (SBIE 2019)

\section{STI - Lógica Livre}

O desenho da arquitetura do STI - Lógica Livre foi inspirado na Arquitetura Tripartida, na qual o sistema é dividido em, pelo menos, três módulos: Módulo do Aluno, Módulo do Domínio e Módulo Tutor (Carbonell, 1970, 1971).

O Módulo do Aluno contém representações do conhecimento do aprendiz referente ao conteúdo. Todas as ações do aluno são utilizadas para simular a sua ZDR (Vygotsky, 1978). O conhecimento do aluno é representado por meio de um grafo direcionado, cujo os nós são as Regras de Equivalência da Lógica Proposicional e os arcos direcionados representam os pré-requisitos da aprendizagem. Os nós contém Pesos que são calculados com base nos erros e acertos (Pontuação) e pela Quantidade de Desafios resolvidos, conforme mostramos na Tabela 1 .

Tabela 1. Regras fuzzy para avaliação do aluno

\begin{tabular}{|c|c|c|c|c|c|}
\hline & Quantidade de Desafios & & Pontuação & & Peso \\
\hline $\mathrm{Se}$ & Pouco & $E$ & Muito Baixa & Logo & Baixo \\
\hline $\mathrm{Se}$ & Pouco & E & Baixa & Logo & Neutro \\
\hline $\mathrm{Se}$ & Pouco & $E$ & Neutra & $\log O$ & Neutro \\
\hline $\mathrm{Se}$ & Pouco & $E$ & Alta & $\log O$ & Neutro \\
\hline $\mathrm{Se}$ & Pouco & $E$ & Muito Alta & Logo & Médio \\
\hline $\mathrm{Se}$ & Razoável & $E$ & Muito Baixa & Logo & Baixo \\
\hline $\mathrm{Se}$ & Razoável & $E$ & Baixa & Logo & Baixo \\
\hline $\mathrm{Se}$ & Razoável & $E$ & Neutra & Logo & Neutro \\
\hline $\mathrm{Se}$ & Razoável & $E$ & Alta & $\log O$ & Médio \\
\hline $\mathrm{Se}$ & Razoável & $E$ & Muito Alta & Logo & Médio \\
\hline $\mathrm{Se}$ & Muito & $E$ & Muito Baixa & Logo & Muito Baixo \\
\hline $\mathrm{Se}$ & Muito & $E$ & Baixa & Logo & Baixo \\
\hline $\mathrm{Se}$ & Muito & $E$ & Neutra & Logo & Neutro \\
\hline $\mathrm{Se}$ & Muito & $E$ & Alta & $\log O$ & Alto \\
\hline $\mathrm{Se}$ & Muito & $E$ & Muito Alta & Logo & Alto \\
\hline
\end{tabular}

As regras representadas pela Tabela 1 passam por um processo de inferência de modo a fazer uma avaliação do conhecimento do aluno sobre determinada Regra de Equivalência. Por exemplo, na regra da linha 5 , se a Quantidade de Desafios é pouco, mesmo que a Pontuação seja Muito Alta, o sistema ainda atribui um peso Médio para o conhecimento do aluno sobre determinada Regra de Equivalência (nó do grafo). Porém, conforme a Quantidade de Desafios resolvidos aumenta, o sistema dá maior ênfase na Pontuação para atribuir o Peso. Assim que o sistema Fuzzy fizer a inferência sobre todas as regras, teremos um grafo com Pesos em cada nó que, por sua vez, constitui a representação do conhecimento do aluno sobre as Regras de Equivalência da Lógica Proposicional.

O conhecimento artificial do STI, por sua vez, está armazenado no Módulo de Domínio, constituindo-se basicamente das Regras de Equivalência. Além da Base de Conhecimento ele contém um motor para resolução de exercícios de Equivalências. Este recebe como entrada uma sequencia de caracteres que supostamente é uma fórmula da Lógica Proposicional e, nesta sequencia, é realizada a Análise Léxica e a Análise Sintática, sendo por fim geradas as fórmulas equivalentes. 
VIII Congresso Brasileiro de Informática na Educação (CBIE 2019)

Anais do XXX Simpósio Brasileiro de Informática na Educação (SBIE 2019)

O Módulo de Domínio contém também uma base de dados pré-carregada com fórmulas lógicas válidas, para que ele possa utilizar delas quando o Módulo Tutor solicitar conteúdos com intuito de formular os desafios. Ademais, o Módulo de Domínio é capaz de calcular quais regras se aplicam em quais fórmulas e o seu respectivo nível de dificuldade, o qual é considerado para propor exercícios para o aluno.

O Módulo Tutor busca fórmulas cujo nível de dificuldade é proporcional ao domínio que o aluno apresenta em determinada regra (Peso no grafo do conhecimento do aluno). Para isso ele utiliza, inclusive, as informações do grafo de representação do conhecimento do aluno, de modo a analisar as regras que o aluno tem domínio (ZDR), para identificar as regras que o aluno está apto a aprender (ZDP).

Além de propor a aplicação de Regras de Equivalência em fórmulas lógicas, o Módulo Tutor também é responsável por prover o feedback da solução apresentada pelo aluno. Caso o aluno erre é apresentada a forma correta de resolver o exercício, mas se o aluno acertar o sistema apresenta uma expressão de felicidade com a solução apresentada.

Os desafios disponibilizados ao aluno são: Selecionar Regras de Equivalência que se aplicam a uma fórmula apresentada; Selecionar Regra de Equivalência que foi aplicada em uma fórmula para gerar outra fórmula; e Digitar a fórmula equivalente dada uma Regra de Equivalência e uma fórmula de origem.

O aluno é incentivado a resolver estes desafios por meio de artifícios que consistem em: desbloquear recursos do sistema, como por exemplo a Calculadora Lógica; incrementar o seu relatório de desempenho; ou até mesmo pela interação com o personagem chamado LoGeek que é mostrado na Figura 1.

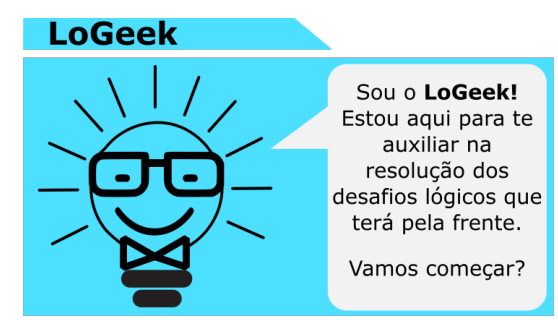

Figura 1. O LoGeeK

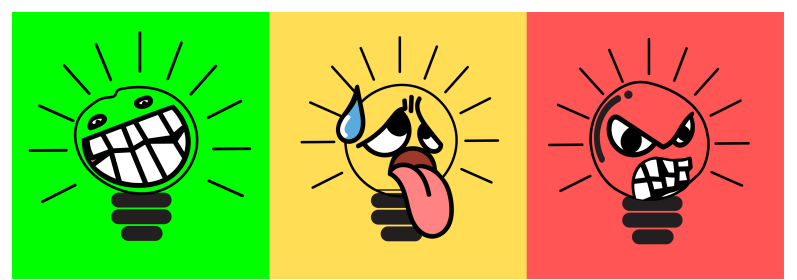

Figura 2. Apresentação do LoGeeK

O personagem LoGeek foi desenvolvido para que o Tutor se mostre mais próximo ao aluno, por isso o LoGeek o acompanha durante o uso do sistema. Ele pode se apresentar de várias formas, demostrando emoções face aos erros e acertos do aluno na resolução dos desafios. Quando o aluno acerta ele é apresentado com semblante feliz e, análogo a isso, aparenta tristeza com o erro, ou então bravo (conforme a relevância do erro), como ilustrado na Figura 2.

Para que não se perca a formalidade de termos técnicos do conteúdo de lógica, $\mathrm{O}$ STI - Lógica Livre utiliza, inclusive, orientações elementares sobre os termos essenciais ao conteúdo, exemplificando conteúdos abstratos de maneira visual. Essas orientações são de uso ilimitado e ficam acessíveis ao aluno durante toda sua interação.

Outro recurso que fica disponível ao aluno é a Calculadora Lógica. Porém constitui-se de um recurso limitado, de modo que sua utilização é liberada na medida em que o aluno resolve os desafios. 
VIII Congresso Brasileiro de Informática na Educação (CBIE 2019)

Anais do XXX Simpósio Brasileiro de Informática na Educação (SBIE 2019)

O STI - Logica Livre é um sistema Web com tela responsiva. Para o seu desenvolvimento foi utilizado, o Bootstrap Framework, que possibilita criação de sites com tela responsiva e com cores atrativas; e o Spring Framework, que possibilita uma conexão direta da linguagem JAVA com uma interface Web

\section{Estudo, Análises e Discussões}

O estudo para validar o STI - Logica Livre como ferramenta educacional foi realizado na disciplina de Introdução a Lógica, no curso de Licenciatura em Computação da UFPR Setor Palotina. Participaram dos experimentos com o sistema um grupo de 21 acadêmicos do primeiro período do curso, no primeiro semestre de 2018. Para verificar se haveria progresso na aprendizagem com a utilização do sistema optou-se por dividir os alunos, de maneira aleatória, em dois grupos, o grupo de estudo e o grupo de apoio.

O grupo de estudo foi constituído pelos alunos que utilizaram a ferramenta educacional, enquanto que o grupo de apoio estudou somente pelos métodos tradicionais, com aulas expositivas e de resolução de exercícios. As seguintes atividades foram realizadas: (1) aplicação do pré-teste; (2) utilização do STI - Lógica Livre pelo grupo de estudo; e (3) aplicação do pós-teste. Todos os alunos realizaram tanto o pré-teste quanto o pós-teste. Vale ressaltar que o nível de complexidade das questões foi maior no pós-teste do que no pré-teste.

Confrontamos as informações de pré-teste e pós-teste de cada grupo em números relativos (ver Figuras 3 e 4), possibilitando assim mensurar o nível de progresso dos alunos.

\begin{tabular}{|c|c|c|}
\hline Grupo Apoio & Total & \\
\hline Aluno_1 & ए & $-10 \%$ \\
\hline Aluno_2 & 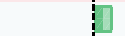 & $8 \%$ \\
\hline Aluno_3 & & $0 \%$ \\
\hline Aluno_4 & 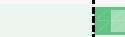 & $15 \%$ \\
\hline Aluno_5 & 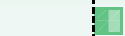 & $13 \%$ \\
\hline Aluno_6 & $\square$ & $-15 \%$ \\
\hline Aluno_7 & 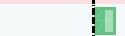 & $10 \%$ \\
\hline Aluno_8 & \begin{tabular}{|l|} 
\\
\end{tabular} & $-41 \%$ \\
\hline Aluno_9 & 口 & $-10 \%$ \\
\hline Aluno_10 & 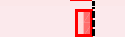 & $-8 \%$ \\
\hline Aluno 11 & $\mathrm{O}$ & $-14 \%$ \\
\hline Total & & $-5 \%$ \\
\hline
\end{tabular}

Figura 3. Grupo de Apoio

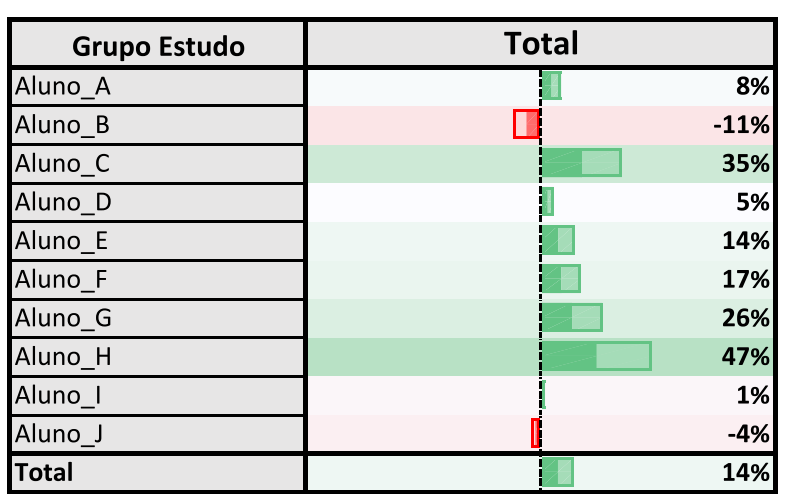

Figura 4. Grupo de Estudo

Mesmo levando em conta que o nível de dificuldade do pós-teste é ligeiramente maior que do pré-teste, destacamos na Figura 3, que: seis dos onze alunos desse grupo apresentaram redução na média de acertos, como por exemplo, o Aluno_8, o qual obteve $80 \%$ de acertos no pré-teste decaindo para $39 \%$ no pós-teste, assim como o aluno_10 que obteve $85 \%$ no pré-teste decaindo para $77 \%$ no pós-teste. Os outros alunos que tiveram resultados inferiores foram os Alunos 1, 6, 9 e 11, com redução no desempenho de $10 \%$, $15 \%, 10 \%$ e $14 \%$, respectivamente. Porém, houve melhoras de até $15 \%$, como é o caso do Aluno_4. A média geral de acertos do grupo de apoio no pré-teste foi de 39\%, decaindo para $34 \%$ no pós-teste. Estes dados representam uma dificuldade na aprendizagem do conteúdo de Equivalência Lógica Proposicional pelo métodos tradicionais de ensino. 
VIII Congresso Brasileiro de Informática na Educação (CBIE 2019)

Anais do XXX Simpósio Brasileiro de Informática na Educação (SBIE 2019)

Análogo a isso, no grupo de estudo (ver Figura 4), temos um cenário diferente. Apenas dois dos dez alunos desse grupo tiveram um desempenho reduzido no pós-teste (Aluno_B decaiu 11\% e o Aluno_J decaiu 4\%). Por outro lado, o Aluno_H que alcançou $47 \%$ de aumento no seu desempenho seguido do Aluno_C com aumento de $35 \%$. Vale resaltar que o grupo de estudos obteve uma melhora significativa no desempenho em relação ao grupo de apoio. O STI - Lógica Livre pode ter contribuído na aprendizagem dos discentes por trazer uma forma de ensino diferenciada e "um-para-um" (Elsom-Cook, 1987).

Além disso, ao analisarmos os $\log s$ de utilização do sistema, verificamos que alguns alunos sequer utilizaram o sistema ou o utilizaram muito pouco, bem como existiram alunos que resolveram centenas de desafios. Para melhor visualização desses dados, fizemos uma correlação com o desempenho individual dos alunos no pré-teste e pós-teste, como vemos na Figura 5. Para representar essa relação utilizamos uma estrutura de gráfico que contém três eixos, um eixo em X, que representa os alunos, e dois eixos em Y: o eixo Y da esquerda (com escala de porcentagem) representa o desempenho no pré-teste e pósteste; já o eixo Y da direita (com escala absoluta) representa a quantidade de desafios que foram resolvidos pelo aluno no STI - Lógica Livre.

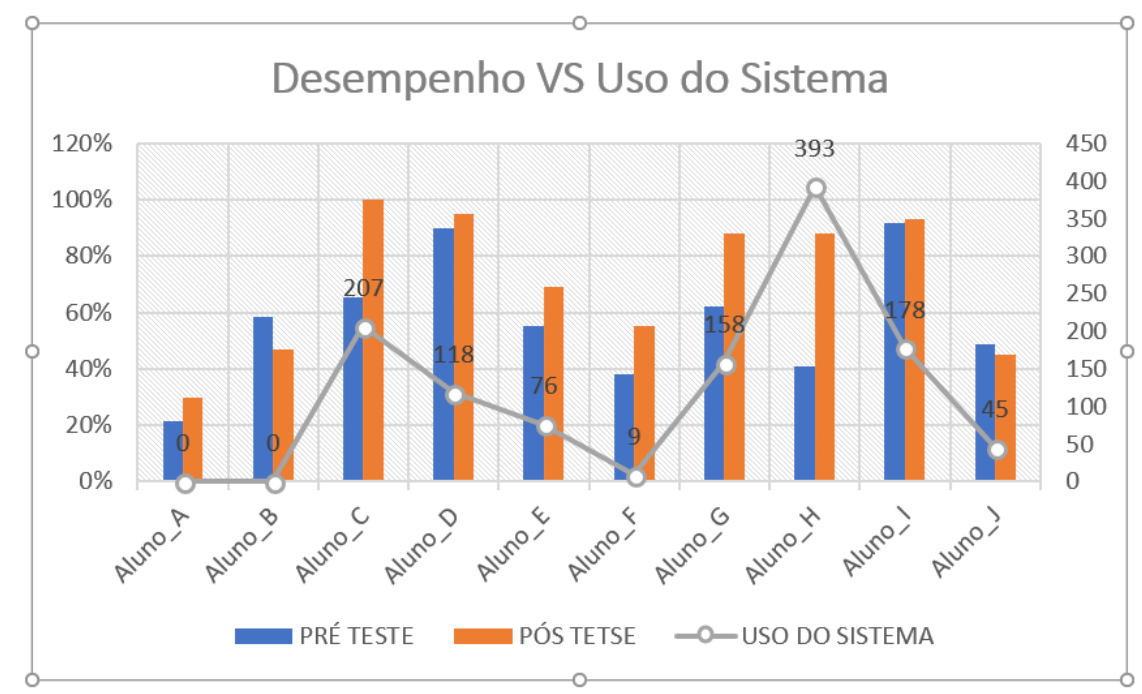

Figura 5. Desempenho $X$ utilização do sistema

Ao analisar o gráfico da figura 5, percebemos uma relação proporcional entre o desempenho dos alunos e a utilização do sistema. Os alunos que mais resolveram desafios tiveram um desempenho maior no pós-teste, como por exemplo, o aluno_C e o aluno_H resolveram, respectivamente, 393 e 207 desafios. Os alunos que menos resolveram desafios tiveram um desempenho inferior no pós-teste, como por exemplo o Aluno_A, Aluno_B, Aluno_F e Aluno_J. Vale ressaltar ainda que os dois únicos alunos que apresentaram desempenho inferior no pós-teste em relação ao pré-teste, foram os alunos $\mathrm{B}$ e $\mathrm{J}$, sendo que o Aluno_B não utilizou o STI - Lógica Livre e o Aluno_J resolveu apenas 45 desafios.

Posteriormente, foi aplicado um questionário com os alunos que se cadastraram no sistema, com intuito de verificar a experiência de uso dos mesmos. Ao questionar sobre a impressão que os alunos tiveram do sistema, todos afirmaram ter uma boa impressão. Sobre os desafios elaborados pelo Tutor, $100 \%$ dos alunos também entendem que foram 
VIII Congresso Brasileiro de Informática na Educação (CBIE 2019)

Anais do XXX Simpósio Brasileiro de Informática na Educação (SBIE 2019)

satisfatórios, atendendo suas expectativa. Vale destacar algumas afirmações dos alunos sobre o sistema, como, "Bem mais fácil que nos livros" e "Foi praticamente $100 \%$ do meu entendimento sobre o conteúdo". Como ponto negativo, foi indicado que o sistema apresentava muitos desafios sobre a mesma Regra de Equivalência.

No geral, a utilização do STI - Lógica Livre pôde contribuir para o processo de aprendizagem de Equivalência Lógica Proposicional dos alunos do curso de Licenciatura em Computação da UFPR - Setor Palotina. Ele pode contribuir para outros alunos de graduação de outras instituições de ensino, desde que estes o utilizem como ferramenta de aprendizagem.

\section{Conclusão}

Por meio deste trabalho foi desenvolvido e utilizado no âmbito da educação superior o STI - Lógica Livre, o qual demostrou ser uma ferramenta eficaz para minimizar as dificuldades de aprendizagem dos alunos no conteúdo de Equivalência Lógica Proposicional. Nossos experimentos realizados no curso de Licenciatura em Computação da UFPR Setor Palotina mostraram que os alunos que de fato utilizaram o sistema tiveram uma melhora nos resultados das avaliações realizadas para validar o STI - Lógica Livre como ferramenta educacional.

Como trabalhos futuros podemos elencar melhorias no sistema, dentre elas: (i) implementar dicas personalizadas, que também levam em conta as características de aprendizagem do aluno; (ii) novas modalidades de desafios para que tenhamos mais mecanismos de aprendizagem; (iii) desenvolver um relatório analítico de desempenho, no qual o aluno possa visualizar os números absolutos de erros e acertos, ou até mesmo rever os desafios e consultar novamente seus feedbacks.

\section{Referências}

Ausubel, D. P. (2003). Aquisição e retenção de conhecimentos: uma perspectiva cognitiva (Vol. 1). Lisboa: Plátano.

Carbonell, J. R. (1970). Ai in cai: An artificial-intelligence approach to computer-assisted instruction. IEEE transactions on man-machine systems, 11(4), 190-202. Retrieved from http://ieeexplore.ieee.org/abstract/ document / 4081977 / ?reload=true

Carbonell, J. R. (1971). Artificial intelligence and large interactive man computer systems. BOLT BERANEK AND NEWMAN INC CAMBRIDGE MASS. Retrieved from http://www.dtic.mil/docs/citations/AD0 726441

Coppin, B. (2010). Inteligência Artificial. Tradução e revisão técnica: Jorge Durães Pires Valério. Rio de Janeiro: LTC.

Elsom-Cook, M. (1987). Intelligent computer-aided instruction research at the open university. cite report no. 10. EUA :Open University: ERIC.

Fowler, D. G. (1991). A model for designing intelligent tutoring systems. Journal of Medical Systems, 15(1), 47-63.

Gavidia, J. J. Z., e ANDRADE, L. C. V. d. (2003). Sistemas tutores inteligentes. Rio de Janeiro-RJ.

Grivokostopoulou, F., Perikos, I., e Hatzilygeroudis, I. (2013). An intelligent tutoring system for teaching fol equivalence. The First Workshop on AI-supported Education for Computer Science (AIEDCS 2013), 20. 
VIII Congresso Brasileiro de Informática na Educação (CBIE 2019)

Anais do XXX Simpósio Brasileiro de Informática na Educação (SBIE 2019)

Klir, G., e Yuan, B. (1995). Fuzzy sets and fuzzy logic (Vol. 4). New Jersey, USA: Prentice hall.

Lodder, J., Heeren, B., e Jeuring, J. (2015). A pilot study of the use of logex, lessons learned. arXiv preprint arXiv:1507.03671. Retrieved from https://arxiv.org/ abs/1507.03671

Lodder, J., Heeren, B., e Jeuring, J. (2016). A domain reasoner for propositional logic. J. UCS, 22(8), 1097-1122. Retrieved from http: / / www • jucs . org/ jucs_22 _8/a_domain_reasoner_for/jucs_22_08_1097_1122_lodder.pdf

Lodder, J., Passier, H., e Stuurman, S. (2008). Using ideas in teaching logic, lessons learned. In Computer science and software engineering, 2008 international conference on (Vol. 5, pp. 553-556).

Mostafavi, B., e Barnes, T. (2017). Evolution of an intelligent deductive logic tutor using data-driven elements. International Journal of Artificial Intelligence in Education, 27(1), 5-36. Retrieved from https://link.springer.com/article/10 $.1007 / \mathrm{s} 40593-016-0112-1$

Nwana, H. S. (1990). Intelligent tutoring systems: an overview. Artificial Intelligence Review, 4(4), 251-277. Retrieved from https://link.springer.com/ article/10.1007/BF00168958

Pimentel, E. P., de França, V. F., e Omar, N. (2003). A identificação de grupos de aprendizes no ensino presencial utilizando técnicas de clusterização. Brazilian Symposium on Computers in Education (Simpósio Brasileiro de Informática na Educação-SBIE), 1(1), 495-504. Retrieved from http://www.br-ie.org/ pub/index.php/sbie/article/view/280

Rissoli, V. R. V., Giraffa, L. M. M., e Martins, J. P. (2006). Sistema tutor inteligente baseado na teoria da aprendizagem significativa com acompanhamento fuzzy. Informática na educação: teoria \& prática, 9(2).

Veen, W., e Vrakking, B. (2009). Homo zappiens: educando na era digital. Porto Alegre : Artimed: Artmed Editora.

Vygotsky, L. S. (1978). Mind in society: The development of higher psychological processes. EUA: Library of congress cataloging in publication data: Harvard university press. 